\title{
Case Report \\ (

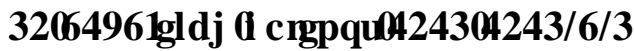 \\ A New Modality for Breast Cancer Diagnosis During the COVID-19 Pandemic. A Case Report
}

\section{Vibert et al. COVID-19, breast cancer and chest scan}

Florence Vibert ${ }^{1}$, Camille Martel ${ }^{1}$, Raluca Andreea Ionescu ${ }^{2}$, Shanti Ame ${ }^{3}$, Carole Mathelin

${ }^{1}$ Department of Surgery, ICANS (Institut de cancérologie Strasbourg Europe), 17 rue Albert Calmette, 67033 Strasbourg Cedex, France

${ }^{2}$ Department of Radiology, Nouvel Hôpital Civil - Hôpitaux Universitaires de Strasbourg, 1 quai Louis Pasteur, 67000 Strasbourg, France

${ }^{3}$ Department of Hemato-oncology, ICANS (Institut de cancérologie Strasbourg Europe), 17 rue Albert Calmette, 67033 Strasbourg Cedex, France

Corresponding Author : Florence Vibert; florence.vibert@chru-strasbourg.fr

Received: 02.04.2021

Accepted: 12.06 .2021

\begin{abstract}
Organized screening for breast cancer (BC) was suspended in most countries of the world during the COVID-19 pandemic. Computed tomography (CT) scans of the chest, frequently performed in patients with severe forms of COVID-19, may detect asymptomatic breast abnormalities. Observation. A 72-year-old patient, with a severe form of COVID-19 benefited
\end{abstract}


from a CT scan. This led to the unexpected discovery, at an early stage, of a $12 \mathrm{~mm}$, high grade, HER-2 positive BC, with a high proliferation index. After responding to chemotherapy, she benefited from conservative breast surgery with sentinel lymph node biopsy. Discussion. Delayed management of BC can be responsible for poor outcomes. Patients with severe forms of COVID-19 are also at risk for developing BC due to common risk factors. Thirty percent of incidental breast lesions discovered on CT scans are unexpected BC. Conclusion. Careful study of the mammary glands on CT scan of patients with COVID-19 may allow early diagnosis of a malignant tumor in a high-risk population for $\mathrm{BC}$ and deprived of routine screening mammography.

Keywords : Breast cancer, COVID-19, SARS-CoV-2, screening, chest scan 


\section{Key Points}

- Breast cancer screening is suspended in many countries around the world during the COVID-19 pandemic.

- Computed tomography scans of the chest are often performed on the patients with severe COVID-19.

- $30 \%$ of breast lesions incidentally discovered on chest scans correspond to cancer.

- Careful study of patients infected with SARS-CoV-2 mammary glands on chest scans may allow early diagnosis of a malignant tumor in a high-risk population for breast cancer and deprived of routine screening mammography. 


\section{Introduction}

SARS-CoV-2 has been responsible for a pandemic that has disrupted the health care system since 2020. Initial publications suggested an increase in the frequency of severe forms in the elderly or those with significant comorbidities (1). Recommendations were modified for the management of patients with breast cancer (BC). The objective was twofold, not to result in a loss of chance from an oncological perspective, while protecting from a possible COVID-19 infection. The suspension of screening was one of these measures $(2,3)$. Computed tomography (CT) scans frequently performed in patients infected with SARS-CoV-2 infections have thus become one of the ways of detecting asymptomatic breast abnormalities.

We report here the case of a patient with high-grade BC diagnosed at an early stage by a CT scan carried out in the context of a SARS-CoV-2 infection, to emphasize the crucial role of chest CT scans.

\section{Observation}

The 72-year-old patient consulted in July 2020 for worsening dyspnea, anosmia and agueusia after a positive PCR test for SARS-CoV-2. The injected chest CT scan findings with multiple bilateral frosted glass ranges with fibrosis were consistent with a SARS-CoV-2 infection (Fig. 1). An unexpected $12 \mathrm{~mm}$ breast mass located in the right breast was highlighted (Fig. 2). Clinical examination of the breast did not reveal a mass and there was no axillary suspicious lymph node (LN). The patient was discharged from the infectious disease department after a 10-day hospitalization.

The mammogram carried out a month later revealed a spiculated mass measuring $12 \mathrm{~mm}$, containing microcalcifications absent a year earlier (Fig. 3a, 3b). Breast ultrasound found a hypoechogenic mass, with irregular contours (Fig. 4). No suspicious LN were visualized. A core needle biopsy confirmed a Grade III infiltrating ductal carcinoma with highly positive 
estrogen and progesterone receptors, overexpression of HER-2, and high proliferation index. A breast MRI confirmed those data. Brain and thoracic-abdominal-pelvic CT scans combined with a bone scan did not reveal any metastases. The multidisciplinary meeting opted for neoadjuvant chemotherapy and trastuzumab, followed by breast conservation surgery with sentinel LN biopsy, radiotherapy and aromatase inhibitors. The pathological examination of the surgical specimen revealed a complete response.

\section{Discussion}

We report here the incidental finding of an early stage $\mathrm{BC}$ through chest imaging carried out as part of the assessment of a SARS-CoV-2 infection.

Interruption of $\mathrm{BC}$ screening during the pandemic had been recommended by many international (3) scientific societies. The rationale for this measure was the potential high risk of SARS-CoV-2 contamination associated with visiting health care centers. Thus, in the absence of a clinical mass, patients did not have access to screening mammograms.

Breast scans present several advantages for diagnosing BC, even in cases of high breast density, without painful manipulation and compression. In this way, its diagnostic performances are improved by contrast product injection (sensibility $90 \%$, 95\% CI $0.785-0.956$; specificity $79 \%, 95 \%$ CI 0.709-0.85) (4). In addition, it is more accessible than MRI. In contrast to breast scans, chest CT scans are not dedicated to studying the mammary gland. However, organs present in scanner sectional drawings such as the two breasts should be studied systematically and carefully.

Late diagnosis of $\mathrm{BC}$ and therefore delayed surgery and treatment may be responsible for worsening tumor stages at diagnosis and decrease survival. Bleicher et al. in 2016 (5) and Mateo et al. in 2019 (6) studied 115,790 and 351,087 patients respectively with invasive, noninflammatory and non-metastatic cancers. They showed a $10 \%$ overall survival decrease per 
additional month between diagnosis and surgical management (95\% CI, 1.07-1.13; $\mathrm{p}<0.001$; 95\% CI, 1.08-1.13; $\mathrm{p}<0.001$, respectively). A large study with more than 420,000 cases confirmed an excess of mortality (HR $1.14,95 \%$ CI 1.09-1.20) in case of surgery performed more than 12 weeks after the initial diagnosis, for stage I (HR 1.19, 95\% CI 1.11-1.28) and stage II (HR 1.16, 95\% CI 1.08-1.25) disease (7). The impact on survival may be related to significant tumor growth, particularly for "aggressive" tumors such as triple negative phenotypes or those overexpressing HER-2. A meta-analysis of 2,533,355 patients (8) showed that delayed surgical management (3 months) resulted in a survival decrease, notably for stage I (HR 1.27, 95\% CI 1.16-1.40) and II cancers (HR 1.13, 95\% CI 1.02-1.24).

Few months after the onset of the COVID-19 pandemic, the impact of screening cessation on tumor characteristics and survival was studied. Vanni et al. (9) found a statistically different LN invasion rate between the 220 patients treated during the pandemic and comparable patients treated a year earlier. $\mathrm{N} 2$ stages were more frequent ( $8 \%$ vs. $2 \%, \mathrm{p}<0.05)$. This locoregional invasion was statistically associated with an extended delay before surgical management (OR 1.07, 95\% CI 1.01-1.13, $\mathrm{p}=0.017)$. Thus, according to the predictions of an English team (10), BC mortality could increase from 7.9 to $9.6 \%$ at 5 years due to a delay in diagnosis.

Given screening interruption, the study of the mammary glands on chest CT scans, very often carried out in patients affected by COVID-19, appears to be essential. People at risk of serious SARS-CoV-2 infections who require further examination or even hospitalization also present an increased risk for BC. Both diseases have common risk factors such as advanced age, obesity, and type 2 diabetes $(3,11,12)$. Chest scans performed as part of the COVID-19 infection check-up are generally not accompanied by administration of iodized contrast except when a pulmonary embolism is suspected, particularly in severe forms (13). However, the injected CT scan is better for exploring the mammary gland. In fact, out of a series of 2,945 patients of all ages who received a chest CT scan, 32 breast lesions were incidentally detected 
(1.1\%) and 29 on injected scans. After further examination, $31 \%$ of the identified lesions were malignant (14). Moyle et al. (15) studied 105,372 scans performed in the general population over a 14-year period. Of the low number of lesions identified $(<1 \%)$, mostly on injected scans (66/78), $28 \%$ were cancerous. The most common cancers identified were invasive carcinomas (14). The lower rate of in situ carcinomas may be explained by the inability to visualize microcalcifications on chest CT scans (15). 


\section{Statement of Ethics}

Written informed consent was obtained from the patient for publication of this case report and any accompanying images.

\section{Declarations of interest}

None.

\section{Fundings Sources}

This research did not receive any specific grand from funding agencies in the public, commercial, or not-for-profit sectors.

\section{Author Contribution}

F.V., C.M., and C.M. were major contributors in writing the manuscript. R.A.I. performed the CT scan. S.A. reviewed English translation. All authors read and approved the final manuscript. 


\section{References}

1. Liang W, Guan W, Chen R, Wang W, Li J, Xu K, et al. Cancer patients in SARS-CoV-2 infection: a nationwide analysis in China. Lancet Oncol. 2020;21(3):335-337. (PMID: 32066541)

2. Gligorov J, Bachelot T, Pierga JY, Antoine EC, Balleyguier C, Barranger E, et al. [COVID-19 and people followed for breast cancer: French guidelines for clinical practice of Nice-St Paul de Vence, in collaboration with the College Nationale des Gynecologues et Obstetriciens Francais (CNGOF), the Societe d'Imagerie de la Femme (SIFEM), the Societe Francaise de Chirurgie Oncologique (SFCO), the Societe Francaise de Senologie et Pathologie Mammaire (SFSPM) and the French Breast Cancer Intergroup-UNICANCER (UCBG)]. Bull Cancer. 2020;107(5):528-537. (PMID: 32278467)

3. Mathelin C, Ame S, Anyanwu S, Avisar E, Mohcen Boubnider W, Breitling K, Ayettey Anie H, Conceição JC, Dupont V, Elder E, Elfgen C, Elonge T, Iglesias E, Imoto S, loannidou-Mouzaka L, A Kappos E, Kaufmann M, Knauer M, Luzuy F, Margaritoni M, Mbodj M, Mundinger A, Orda R, Ostapenko V, Özbaş S, Özmen V, Pagani O, Pieńkowski T, Schneebaum S, Shmalts E, Selim E, Pavel Z, Lodi M, Maghales-Costa M. Breast Cancer Management During the COVID-19 Pandemic: The Senologic International Society Survey. Eur J Breast Health. 2021;17(2):188196.

4. Uhlig J, Uhlig A, Biggemann L, Fischer U, Lotz J, Wienbeck S. Diagnostic accuracy of cone-beam breast computed tomography: a systematic review and diagnostic meta-analysis. Eur Radiol. 2019;29(3):1194-1202. (PMID: 30255249)

5. Bleicher RJ, Ruth K, Sigurdson ER, Beck JR, Ross E, Wong YN, et al. Time to Surgery and Breast Cancer Survival in the United States. JAMA Oncol. 2016;2(3):330-339. (PMID: 26659430)

6. Mateo AM, Mazor AM, Obeid E, Daly JM, Sigurdson ER, Handorf EA, et al. Time to Surgery and the Impact of Delay in the Non-Neoadjuvant Setting on Triple-Negative Breast Cancers and Other Phenotypes. Ann Surg Oncol. 2020;27(5):1679-1692. (PMID: 31712923)

7. Polverini AC, Nelson RA, Marcinkowski E, Jones VC, Lai L, Mortimer JE, et al. Time to Treatment: Measuring Quality Breast Cancer Care. Ann Surg Oncol. 2016;23(10):3392-3402. (PMID: 27503492)

8. Johnson BA, Waddimba AC, Ogola GO, Fleshman JW, Jr., Preskitt JT. A systematic review and meta-analysis of surgery delays and survival in breast, lung and colon cancers: Implication for surgical triage during the COVID-19 pandemic. Am J Surg. 2020 Dec 8. doi: 10.1016/j.amjsurg.2020.12.015. [Epub ahead of print]. (PMID: 33317814)

9. Vanni G, Tazzioli G, Pellicciaro M, Materazzo M, Paolo O, Cattadori F, et al. Delay in Breast Cancer Treatments During the First COVID-19 Lockdown. A Multicentric Analysis of 432 Patients. Anticancer Res. 2020;40(12):7119-7125. (PMID: 33288611)

10. Maringe C, Spicer J, Morris M, Purushotham A, Nolte E, Sullivan R, et al. The impact of the COVID-19 pandemic on cancer deaths due to delays in diagnosis in England, UK: a national, population-based, modelling study. Lancet Oncol. 2020;21(8):1023-1034. (PMID: 32702310)

11. Momenimovahed Z, Salehiniya H. Epidemiological characteristics of and risk factors for breast cancer in the world. Breast Cancer (Dove Med Press). 2019;11:151-164. (PMID: 31040712)

12. Seiglie J, Platt J, Cromer SJ, Bunda B, Foulkes AS, Bassett IV, et al. Diabetes as a Risk Factor for Poor Early Outcomes in Patients Hospitalized With COVID-19. Diabetes Care. 2020;43(12):2938-2944. (PMID: 32847827)

13. Jalaber C, Lapotre T, Morcet-Delattre T, Ribet F, Jouneau S, Lederlin M. Chest CT in COVID-19 pneumonia: A review of current knowledge. Diagn Interv Imaging. 2020;101(7-8):431-437. (PMID: 32571748)

14. Monzawa S, Washio T, Yasuoka R, Mitsuo M, Kadotani Y, Hanioka K. Incidental detection of clinically unexpected breast lesions by computed tomography. Acta Radiol. 2013;54(4):374379. (PMID: 23395815)

15. Moyle $\mathrm{P}$, Sonoda L, Britton $\mathrm{P}$, Sinnatamby R. Incidental breast lesions detected on $\mathrm{CT}$ : what is their significance? Br J Radiol. 2010;83(987):233-240. (PMID: 19546179) 
Figures

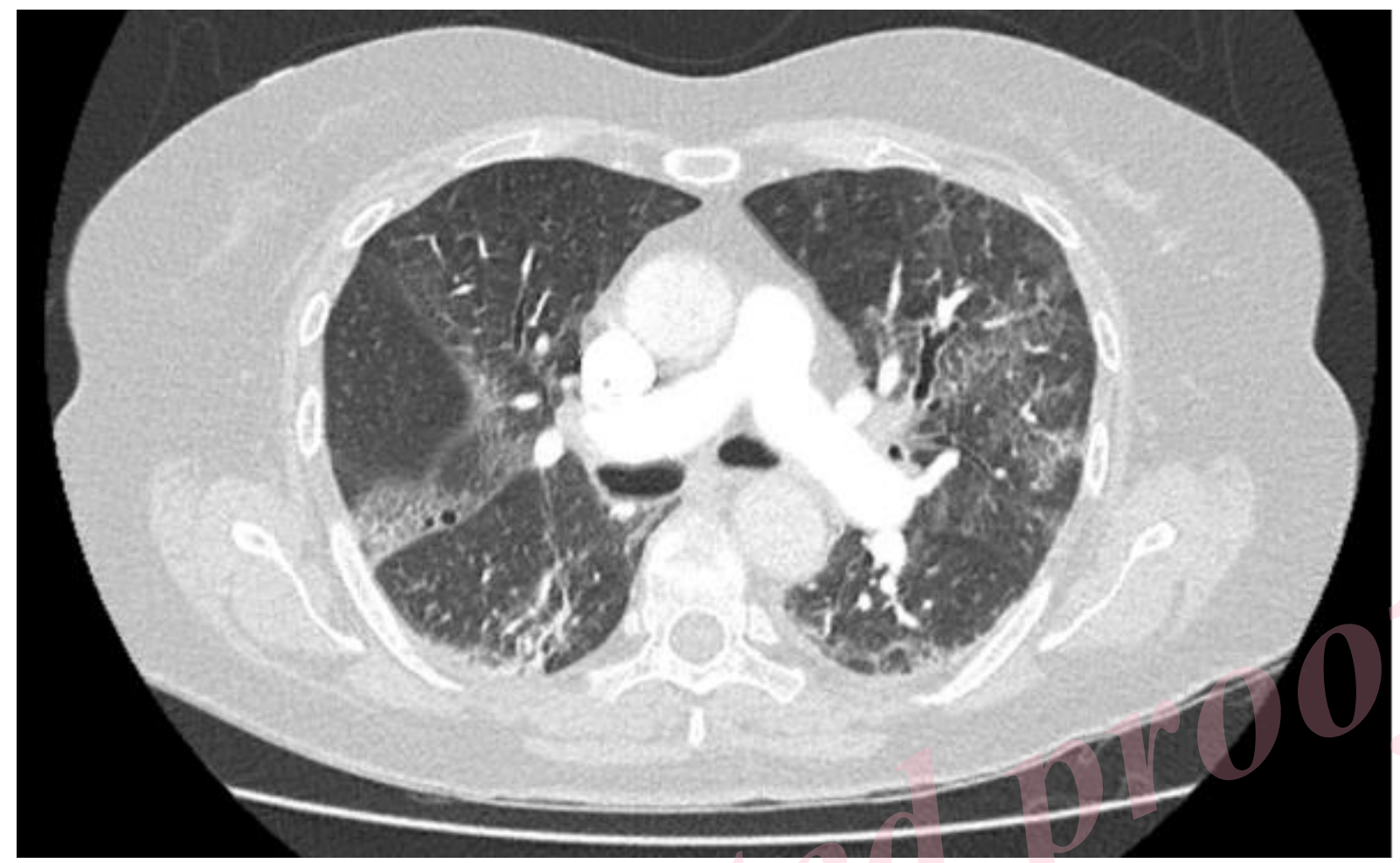

Fig. 1. Chest CT scan with multiple bilateral frosted glass ranges with fibrosis.

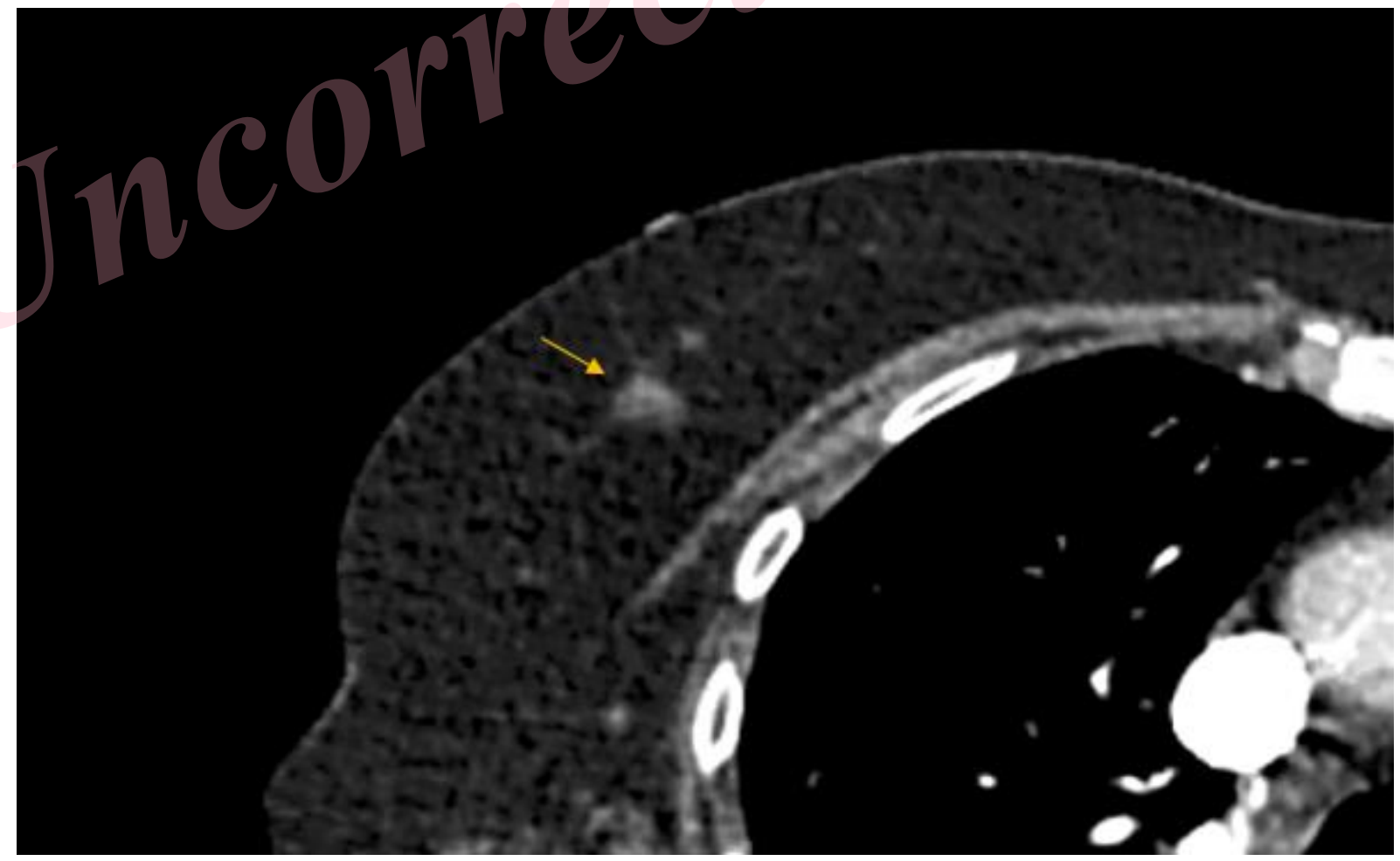

Fig. 2. An unexpected right breast mass of $12 \mathrm{~mm}$ on chest CT scan. 


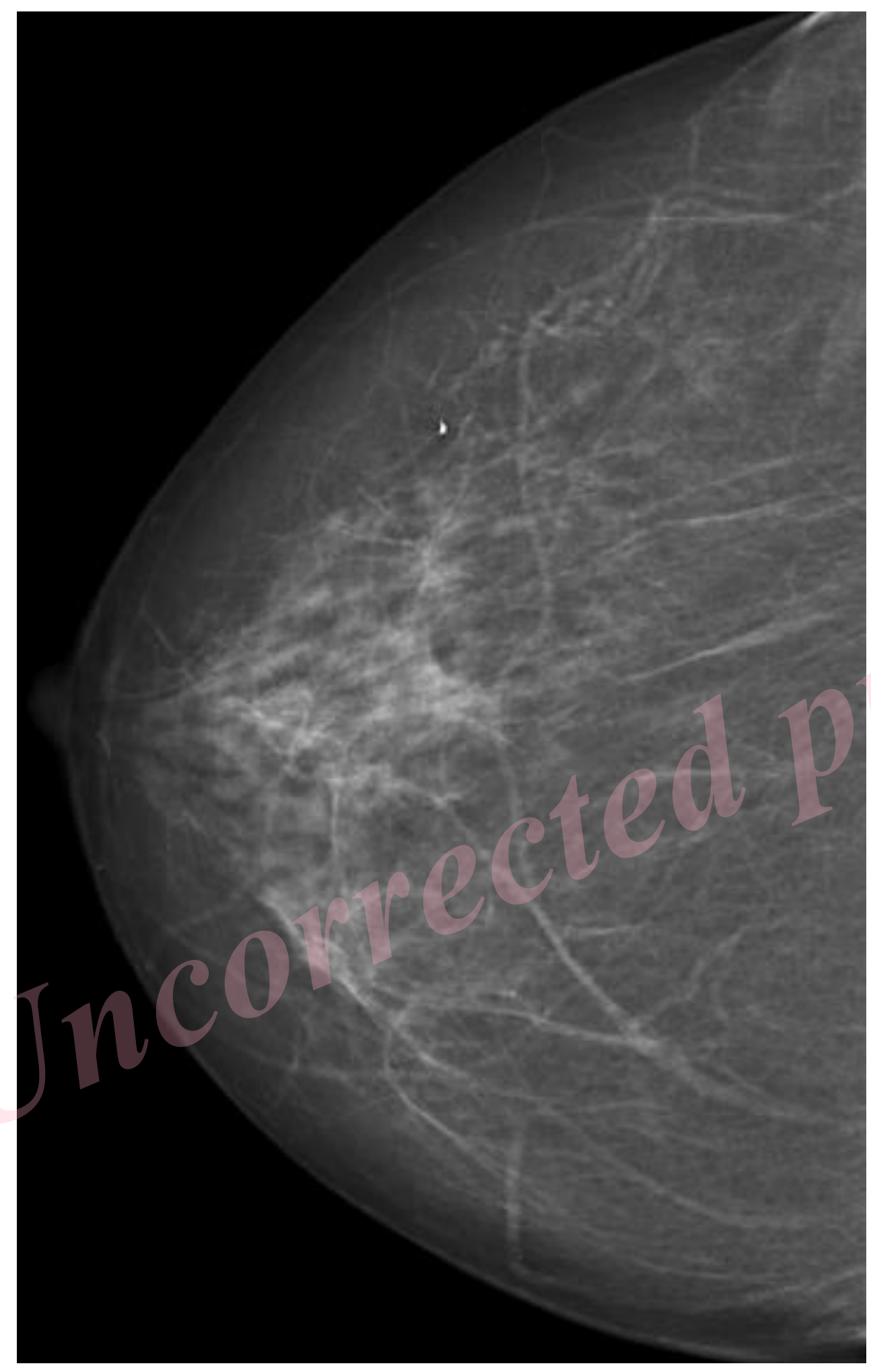

Fig. 3a. The mass was seen on the mammogram (frontal) as a dense, poorly limited, spiculated mass, containing microcalcifications. 


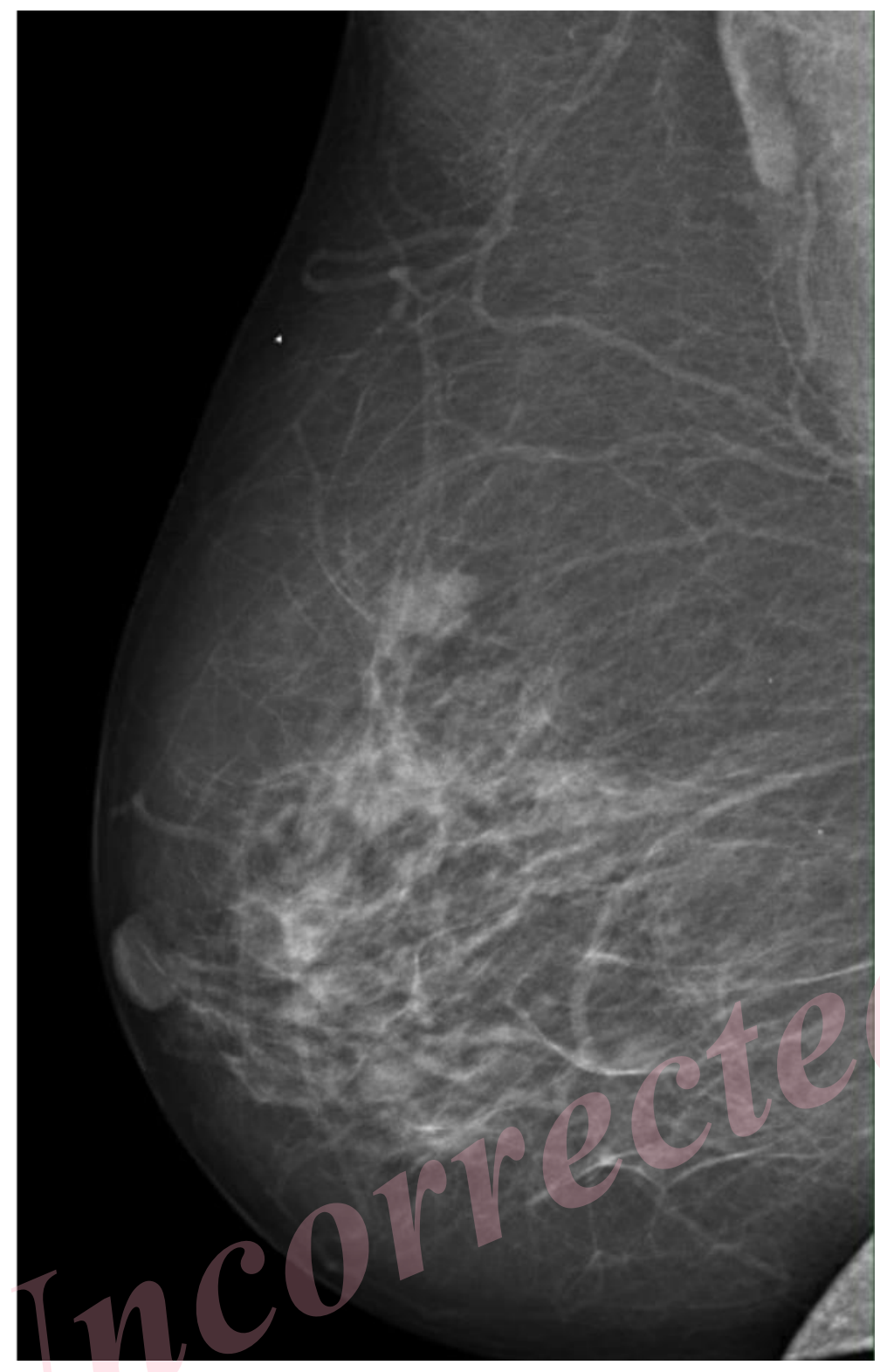

Fig. 3b. The mass was seen on the mammogram (lateral) as a dense, poorly limited, spiculated mass, containing microcalcifications. 


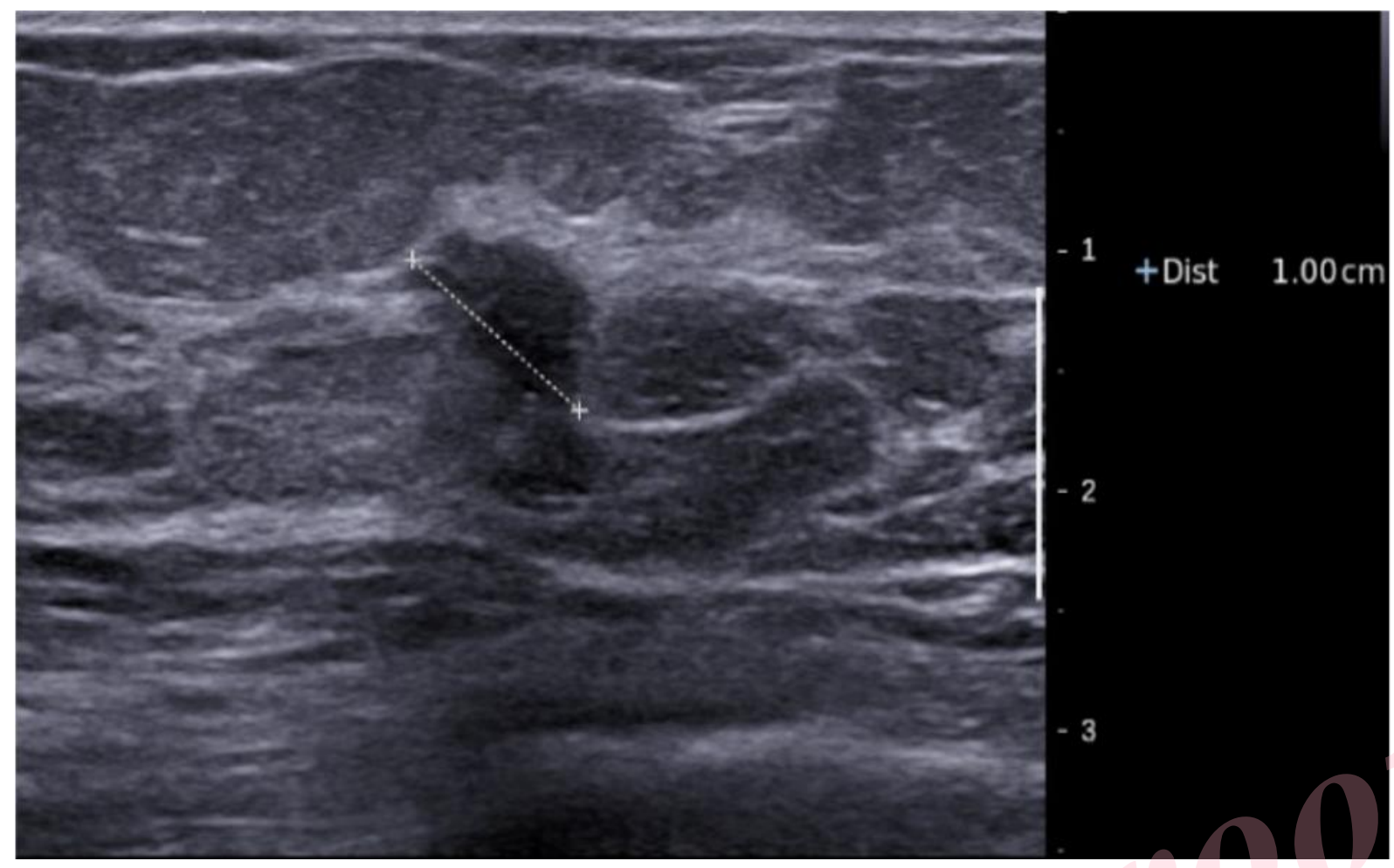

Fig. 4. The breast ultrasound found a hypoechogenic mass, with irregular contours and a large vertical axis. 